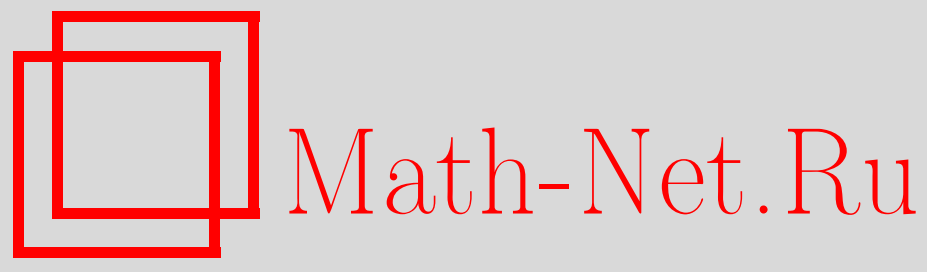

О. В. Кузьмин, О. В. Леонова, О полиномах разбиений, Дискрет. матем., 2001, том 13, выпуск 2, 144-158

DOI: https://doi.org/10.4213/dm283

Использование Общероссийского математического портала Math-Net.Ru подразумевает, что вы прочитали и согласны с пользовательским соглашением http://www . mathnet.ru/rus/agreement

Параметры загрузки:

IP : 54.198 .187 .58

26 апреля 2023 г., $11: 52: 25$

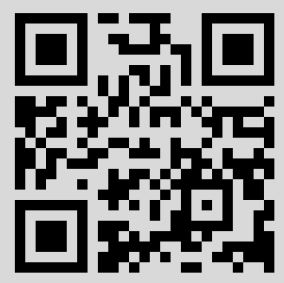


УДК 519.1

\section{О полиномах разбиений}

(C) 2001 г. . В. Кузьмин, О. В. Леонова

Рассматриваются некоторые из полиномов разбиений: однородные полиномы Белла и Платонова, обобщенные $A$ - и $B$-полиномы, $T$ - и $C$-полиномы Тушара и $P$-полиномы, составляющие с $T$-полиномами квазиортогональную систему. Для всех указанных полиномов изучаются коэффициенты, для которых приводятся рекуррентные соотношения и перечислительные интерпретации. Для $C$-полиномов найдены рекуррентные соотношения, производящие функции и явное представление.

\section{1. Введение}

Понятие полинома разбиений, полинома от нескольких переменных, определяемого с помощью суммы по различным разбиениям значений его индекса, введено Беллом в [1]. Один из таких полиномов, связанный с производными от композиции функций, в [2] назван полиномом Белла. Ряд свойств коэффициентов $n$-го полинома Белла, так называемых однородных полиномов Белла $A_{n, k}(x)$, и сопряженных с ними полиномов Платонова $B_{n, k}(x)$ приведен в [3].

Обобщенными $A$ - и $B$-полиномами $A_{n k}^{(m)}(x)$ и $B_{n k}^{(m)}(x)$ соответственно в [4] названы обобщения полиномов $A_{n k}(x)$, которые имеются, например, в [5], [6], и обобщения полиномов, введенные в [7]. Ряд свойств обобщенных $A$ - и $B$-полиномов установлен B [8].

В связи с изучением некоторых свойств цикловой структуры подстановок Тушар в [9] ввел ряд обобщений полиномов Белла. Для одного класса таких полиномов $T_{n, k}(x, y)$, названных полиномами Тушара, в [10] получены экспоненциальные производящие функции и рекуррентные соотношения. В [11] рассматриваются свойства некоторых частных случаев полиномов $T_{n, k}(x, y)$ и вновь введенных полиномов $\mathrm{Ty}-$ шара $C_{n, k}(x, y)$. Полученные формулы используются в задачах о случайных блужданиях.

В $[12,13]$ авторами данной публикации изучаются полиномы $T_{n, k}(x, y)$ и $C_{n, k}(x, y)$, при этом получены обобщения некоторых из приведенных в [3] свойств полиномов $A_{n, k}(x)$, перечислительные интерпретации и вероятностные приложения. В [14] вводятся новые, так называемые $P$-полиномы, составляющие с $T$-полиномами Тушара квазиортогональную систему. Для $P$-полиномов $P_{n, k}(x, y)$ получено явное выражение, производящие функции и рекуррентные соотношения, построены алгоритм преобразования $T$-полиномов в $P$-полиномы и ему обратный.

Во втором параграфе статьи приводятся необходимые определения и свойства полиномов Белла и Платонова и их коэффициентов. 
Во третьем параграфе приводятся необходимые определения и свойства обобщенных $A$ - и $B$-полиномов и изучаются их коэффициенты.

В четвертом параграфе рассматриваются $T$ - и $C$-полиномы Тушара и $P$-полиномы. Изучаются коэффициенты указанных полиномов, приводятся рекуррентные соотношения и перечислительные интерпретации. Для $C$-полиномов найдены рекуррентные соотношения, производящие функции и явное представление.

\section{2. $A$ - и $B$-полиномы}

\section{1. Однородные полиномы Белла}

Однородные полиномы Белла от переменных $x_{i}, i \geqslant 1$, которые мы будем обозначать $A_{n, k}(x)=A\left(n, k ; x_{1}, x_{2}, \ldots\right), 1 \leqslant k \leqslant n$, и в дальнейшем называть $A$-полиномами, впервые рассматривались Беллом в [1]. Пусть последовательность аргументов $A$ полиномов задана в виде производящей функции

$$
x(t)=\sum_{n=1}^{\infty} x_{n} \frac{t^{n}}{n !} .
$$

Производящими функциями $A$-полиномов являются

$$
\begin{aligned}
\sum_{n=k}^{\infty} A_{n, k}(x) \frac{t^{n}}{n !} & =\frac{(x(t))^{k}}{k !}, \\
\sum_{k=0}^{\infty} \sum_{n=k}^{\infty} A_{n, k}(x) \frac{g^{k} t^{n}}{n !} & =e^{g x(t)}
\end{aligned}
$$

Из (1) или (2) может быть получена явная формула для $A$-полиномов

$$
A_{n, k}(x)=n ! \sum_{n, k} \prod_{i=1}^{n-k+1} x_{i}^{r_{i}}\left(r_{i} !(i !)^{r_{i}}\right)^{-1}
$$

где $A_{0,0}(x)=1$ и суммирование ведется по всем $r_{i} \geqslant 0,1 \leqslant i \leqslant n$, таким, что

$$
\sum_{i=1}^{n-k+1} r_{i}=k, \quad \sum_{i=1}^{n-k+1} i r_{i}=n
$$

Пусть

$$
\mathscr{D}_{x}=x_{2} \frac{\partial}{\partial x_{1}}+x_{3} \frac{\partial}{\partial x_{2}}+\ldots
$$

Для $A$-полиномов известна рекуррентная формула (см., например, [2])

$$
A_{n, k}(x)=x_{1} A_{n-1, k-1}(x)+\mathscr{D}_{x} A_{n-1, k}(x), \quad n, k \geqslant 1, \quad k \leqslant n .
$$

Очевидно, что при каждом произведении $\prod_{i=1}^{n-k+1} x_{i}^{r_{i}}$ коэффициент в (3) зависит от спецификации соответствующего разбиения

$$
\sum_{i=1}^{n-k+1} r_{i}=k, \quad \sum_{i=1}^{n-k+1} i r_{i}=n .
$$


Положим $r=\left(r_{1}, r_{2}, \ldots, r_{n-k+1}\right)$, тогда (см. [15])

$$
A_{n, k}(x)=\sum_{n, k} a_{n, k}(r) \prod_{i=1}^{n-k+1} x_{i}^{r_{i}}, \quad 1 \leqslant k \leqslant n
$$

где

$$
a_{n, k}(r)=n ! \prod_{i=1}^{n-k+1}\left(r_{i} !(i !)^{r_{i}}\right)^{-1}
$$

Выражение (5) показывает, что коэффициент $a_{n, k}(r)$ равен числу подстановок степени $n$, состоящих из $k$ упорядоченных циклов, из которых $r_{i}$ имеют длину $i$, $1 \leqslant i \leqslant n-k+1$.

Сравнивая коэффициенты в левой и правой частях (4), стоящие при произведении $\prod_{i=1}^{n-k+1} x_{i}^{r_{i}}$, получим (см. [15]) равенство

$$
\begin{aligned}
& a_{n, k}(r)=a_{n-1, k-1}\left(r_{1}-1, r_{2}, \ldots, r_{n-k+1}\right) \\
& \quad+\sum_{i=1}^{n-k}\left(r_{i}+1\right) a_{n-1, k}\left(r_{1}, \ldots r_{i-1}, r_{i}+1, r_{i+1}-1, r_{i+2}, \ldots, r_{n-k+1}\right) .
\end{aligned}
$$

Известно, что числа Стирлинга и Лаха (см. например [2]) могут быть представлены через коэффициенты $a_{n, k}(r)$ в следующем виде [15]:

$$
\begin{aligned}
S(n, k) & =\sum_{n, k} a_{n, k}(r) \\
|s(n, k)| & =\sum_{n, k} a_{n, k}(r) \prod_{i=1}^{n-k+1}((i-1) !)^{r_{i}} \\
|L(n, k)| & =\sum_{n, k} a_{n, k}(r) \prod_{i=1}^{n-k+1}(i !)^{r_{i}}
\end{aligned}
$$

где $n \geqslant 2,1 \leqslant k \leqslant n-1$ и $s(n, k), S(n, k)$ и $L(n, k)$ - числа Стирлинга первого и второго рода и числа Лаха соответственно.

\section{2. Полиномы Платонова}

C $A$-полиномами тесно связаны $B$-полиномы Платонова, введенные в [16] и более систематично описанные в книге [17]. Строго говоря, это полиномы только от переменньх $x_{i}, i \geqslant 2$, роль переменной $x_{1} \neq 0$ нормирующая. Для $B$-полиномов справедливо явное выражение

$$
B_{n, k}(x)=\frac{(-1)^{n-k}}{(k-1) ! x_{1}^{2 n-k}} \sum_{2 n-2 k, n-k}(-1)^{r_{1}} r_{1} !\left(2 n-k-r_{1}-1\right) ! \prod_{i=1}^{n-k+1} x_{i}^{r_{i}}\left(r_{i} !(i !)^{r_{i}}\right)^{-1},
$$

где $n \geqslant 2,1 \leqslant k \leqslant n-1, B_{n, n}=x_{1}^{-n}$ при $n \geqslant 0$ и суммирование ведется по всем $r_{i} \geqslant 0,1 \leqslant i \leqslant 2 n-2 k$, таким, что

$$
\sum_{i=1}^{n-k+1} r_{i}=n-k, \quad \sum_{i=1}^{n-k+1} i r_{i}=2 n-2 k
$$


Тесная связь $A$ - и $B$-полиномов объясняется тем, что они двояко, алгебраически и аналитически, сопряжены. Алгебраическая сопряженность определяется взаимной обратностью бесконечных нижних треугольных матриц $\left\|A_{n, k}(x)\right\|$ и $\left\|B_{n, k}(x)\right\|$. Аналитическая сопряженность выражается системой тождеств

$$
A_{n, k}(x)=B_{n, k}(\tilde{x})
$$

где $x(t)$ и $\tilde{x}(u)$ - взаимно обратные функции, то есть

$$
x(\tilde{x}(u))=u, \quad \tilde{x}(x(t))=t .
$$

С учетом соотношения (8) из (1) и (2) получаем, что производящими функциями для $B$-полиномов являются

$$
\begin{aligned}
\sum_{n=k}^{\infty} B_{n, k}(x) \frac{u^{n}}{n !} & =\frac{\tilde{x}(u)^{k}}{k !} \\
\sum_{k=0}^{\infty} \sum_{n=k}^{\infty} B_{n, k}(x) \frac{g^{k} u^{n}}{n !} & =e^{g \tilde{x}(u)}
\end{aligned}
$$

Для $B$-полиномов справедливо (см. [17]) рекуррентное соотношение

$$
x_{1} B_{n, k}(x)=B_{n-1, k-1}(x)+\mathscr{D}_{x} B_{n-1, k}(x), \quad n, k \geqslant 1, \quad k \leqslant n .
$$

Выше уже отмечалось, что $B_{n, k}(x)$ являются полиномами только от переменных $x_{i}$, $i \geqslant 2$. Вводя нормирующие множители вида $(-1)^{n-k} x_{1}^{2 n-k}$, получим полиномы от всех переменных $x_{i}, i \geqslant 1$, вида

$$
B_{n, k}^{\prime}(x)=(-1)^{n-k} x_{1}^{2 n-k} B_{n, k}(x)=\sum_{2 n-2 k, n-k} b_{n, k}(r) \prod_{i=1}^{n-k+1} x_{i}^{r_{i}}
$$

где

$$
b_{n, k}(r)=\frac{(-1)^{r_{1}} r_{1} !\left(2 n-k-r_{1}-1\right) !}{(k-1) !} \prod_{i=1}^{n-k+1}\left(r_{i} !(i !)^{r_{i}}\right)^{-1} .
$$

Как видно из выражения (11), величина

$$
b_{n, k}(r) \frac{(-1)^{r_{1}}(k-1) !}{r_{1} !\left(2 n-k-r_{1}-1\right)_{k-r_{1}-1}}
$$

равна числу подстановок степени $2 n-2 k$, состоящих из $n-k$ упорядоченных циклов, из которых $r_{i}$ имеют длину $i, 1 \leqslant i \leqslant n-k+1$.

Сравнивая в левой и правой частях формулы (10) коэффициенты при произведении $\prod_{i=1}^{n-k+1} x_{i}^{r_{i}}$, находим, что

$$
\begin{aligned}
b_{n, k}(r) & =b_{n-1, k-1}(r)+(k-2 n+2) b_{n-1, k}\left(r_{1}, r_{2}-1, r_{3}, \ldots, r_{n-k+1}\right) \\
- & \sum_{i=1}^{n-k}\left(r_{i}+1\right) b_{n-1, k}\left(r_{1}-1, r_{2}, \ldots, r_{i-1}, r_{i}+1, r_{i+1}-1, r_{i+2}, \ldots, r_{n-k+1}\right) .
\end{aligned}
$$


Числа Стирлинга и Лаха могут быть представлены через коэффициенты $b_{n, k}(r)$ в виде [15]

$$
\begin{aligned}
S(n, k) & =\sum_{2 n-2 k, n-k} b_{n, k}(r) \prod_{i=1}^{n-k+1}((i-1) !)^{r_{i}}, \\
|s(n, k)| & =\sum_{2 n-2 k, n-k} b_{n, k}(r), \\
|L(n, k)| & =\sum_{2 n-2 k, n-k} b_{n, k}(r) \prod_{i=1}^{n-k+1}(i !)^{r_{i}},
\end{aligned}
$$

где $n \geqslant 2,1 \leqslant k \leqslant n-1$.

\section{3. Обобщенные $A$ - и $B$-полиномы}

\section{1. Обобщенные $A$-полиномы}

Обобщенные $A$-полиномы $A_{n k}^{(m)}(x)$ для фиксированного целого неотрицательного $m$ могут быть определены как коэффициенты в суммах

$$
\sum_{n=k(m+1)}^{\infty} \frac{A_{n k}^{(m)}(x)}{n !} t^{n}=\frac{1}{k !}\left(\sum_{i=m+1}^{\infty} \frac{x_{i} t^{i}}{i !}\right)^{k}
$$

где $x_{m+1} \neq 0, k \geqslant 1$. Известно, что $A_{n k}^{(0)}(x)=A_{n k}(x)$.

Как видно из (3), для $n \geqslant 1,0 \leqslant k<(n+m+1) /(m+1)$, явное выражение для полиномов $A_{n k}^{(m)}(x)$ имеет вид

$$
A_{n k}^{(m)}(x)=n ! \sum_{n-k m, k} \prod_{i=1}^{n-k(m+1)+1} x_{i+m}^{r_{i}}\left(r_{i} !((i+m) !)^{r_{i}}\right)^{-1}
$$

где $x=\left(x_{m+1}, x_{m+2}, \ldots\right)$ - формальные переменные, а суммирование ведется по всем разбиениям натурального числа $n-k m$ на $k$ натуральных не меньших $m$ слагаемых.

Полиномы $A_{n k}^{(m)}(x)$ удовлетворяют соотношениям [7]

$$
A_{n+1, k}^{(m)}(x)=\left(\begin{array}{c}
n \\
m
\end{array}\right) x_{m+1} A_{n-m, k-1}^{(m)}(x)+\sum_{i=m+1}^{n-k+1} x_{i+1} \frac{\partial}{\partial x_{i}} A_{n k}^{(m)}(x),
$$

где $1 \leqslant k \leqslant[(n+1) /(m+1)], n \geqslant m$.

Соотношение (13) можно переписать в виде

$$
A_{n, k}^{(m)}(x)=\sum_{n-k m, k} a_{n, k}^{(m)}(r) \prod_{i=1}^{n-k(m+1)+1} x_{i+m}^{r_{i}}
$$


где

$$
a_{n, k}^{(m)}(r)=n ! \prod_{i=1}^{n-k(m+1)+1}\left(r_{i} !((i+m) !)^{r_{i}}\right)^{-1} .
$$

Выражение (15) показывает, что коэффициент $a_{n, k}^{(m)}(r)$ равен числу подстановок степени $n$, состоящих из $k$ упорядоченных циклов, из которых $r_{i}$ имеют длину $i+m$, $1 \leqslant i \leqslant n-k(m+1)+1$.

Сравнивая в формуле (14) коэффициенты в правой и левой частях при произведении $\prod_{i=1}^{n-k(m+1)+1} x_{i}^{r_{i}}$, получаем рекуррентное соотношение

$$
\begin{aligned}
a_{n+1, k}^{(m)}(r)= & \left(\begin{array}{c}
n \\
m
\end{array}\right) a_{n-m, k-1}^{(m)}\left(r_{1}, \ldots, r_{m+1}-1, \ldots, r_{n-k(m+1)+1}\right) \\
& +\sum_{i=m+1}^{n-k(m+1)+1}\left(r_{i}+1\right) a_{n, k}^{(m)}\left(r_{1}, \ldots, r_{i}+1, r_{i+1}-1, \ldots, r_{n-k(m+1)+1}\right) .
\end{aligned}
$$

Полагая $m=0$ в соотношении (16), получаем соотношение (6).

Отметим, что $m$-присоединенные числа Стирлинга (см., например [5]) представимы через коэффициенты $a_{n, k}^{(m)}(r)$ в виде

$$
\begin{aligned}
{ }^{m+1} s(n, k) & =\sum_{n, k} a_{n, k}^{(m)}(r) \prod_{i=1}^{n-k+1}((i-1) !)^{r_{i}}, \\
{ }^{m+1} S(n, k) & =\sum_{n, k} a_{n, k}^{(m)}(r), \quad n \geqslant 2, \quad 1 \leqslant k \leqslant n-1,
\end{aligned}
$$

где ${ }^{m} s(n, k)$ и ${ }^{m} S(n, k)-m$-присоединенные числа Стирлинга первого и второго рода соответственно.

Полагая $m=0$ в соотношении (17) получаем первое и второе соотношения (7).

\section{2. Обобщенные $B$-полиномы}

Обобщенные $B$-полиномы $B_{n k}^{(m)}(x)$ для фиксированного натурального $m$ задаются [7] соотношениями

где

$$
B_{n k}^{(m)}(x)=\left(\begin{array}{l}
n-1 \\
k-1
\end{array}\right) \frac{d^{n-k}}{d t^{n-k}}\left[\frac{x(t)}{t^{m}}\right]_{t=0}^{-n / m}
$$

$$
x(t)=\sum_{i=m}^{\infty} x_{i} \frac{t^{i}}{i !}, \quad x_{m} \neq 0, \quad n \geqslant 1, \quad 1 \leqslant k \leqslant n .
$$

Известно, что $B_{n k}^{(1)}(x)=B_{n k}(x)$.

В [7] показано, что при $n \geqslant 2,1 \leqslant k \leqslant n-1, x_{m} \neq 0$

$$
\begin{aligned}
B_{n k}^{(m)}(x) & =\frac{(n-1) !}{(k-1) !}(-1)^{n-k}\left(\frac{m !}{x_{m}}\right)^{n / m+n-k} \\
& \times \sum_{2 n-2 k, n-k}(-1)^{r_{1}} r_{1} ! \prod_{j=0}^{n-k-r_{1}-1}\left(\frac{n}{m}+j\right) \prod_{i=1}^{n-k+1} x_{i+m-1}^{r_{i}}\left(r_{i} !((i+m-1) !)^{r_{i}}\right)^{-1}
\end{aligned}
$$


где суммирование ведется по всем разбиениям натурального числа $2 n-2 k$ на $n-k$ натуральных слагаемьх. Дополнительно полагают, что

$$
B_{n n}^{(m)}(x)=\left(x_{m}^{-1} m !\right)^{n / m}, \quad n \geqslant 1
$$

Обобщенные $B$-полиномы удовлетворяют рекуррентному соотношению (см. [18])

$$
B_{n+m, k+m-1}^{(m)}(x)=\frac{m !(n+m-1)_{m-1}}{x_{m}(k+m-2)_{m-1}}\left(B_{n, k-1}^{(m)}(x)+\mathscr{L} B_{n k}^{(m)}(x)\right)
$$

где

$$
\mathscr{L}=x_{m+1} \partial / \partial x_{m}+x_{m+2} \partial / \partial x_{m+1}+\ldots
$$

Вводя нормирующие множители вида $(-1)^{n-k} x_{m}^{n-k+n / m}$, получим полиномы от всех переменных $x_{i}, i \geqslant m$, вида

$$
\hat{B}_{n, k}^{(m)}(x)=(-1)^{n-k} x_{m}^{n-k+n / m} B_{n, k}^{(m)}(x)=\sum_{2 n-2 k, n-k} b_{n, k}^{(m)}(r) \prod_{i=1}^{n-k+1} x_{i+m}^{r_{i}}
$$

где

$$
\begin{aligned}
b_{n, k}^{(m)}(r)=\frac{(-1)^{r_{1}} r_{1} !(n-1) !(m !)^{n-k+n / m}}{(k-1) ! m^{n-k-r_{1}}} & \times \prod_{j=0}^{n-k-r_{1}-1}(n+j m) \prod_{i=1}^{n-k+1}\left(r_{i} !((i+m-1) !)^{r_{i}}\right)^{-1} .
\end{aligned}
$$

Как видно из выражения (19),

$$
b_{n, k}^{(m)}(r) \frac{(n-1) !}{(k-1) !(2 n-2 k) !}(-1)^{r_{1}} r_{1} !(m !)^{n-k+n / m} \prod_{j=0}^{n-k-r_{1}-1}\left(\frac{n}{m}+j\right)
$$

равно числу подстановок степени $2 n-2 k$, состоящих из $n-k$ упорядоченных циклов, из которых $r_{i}$ имеют длину $i, m \leqslant i \leqslant n$.

Сравнивая в (18) коэффициенты в левой и правой частях при произведении $\prod_{i=1}^{n-k+1} x_{i}^{r_{i+m}}$, получим рекуррентное соотношение

$$
\begin{aligned}
& b_{n+m, k+m-1}^{(m)}(r)=\frac{m !(n+m-1)_{m-1}}{(k+m-2)_{m-1}} \\
& \quad \times\left(b_{n, k-1}^{(m)}(r)-(k-n-n / m) b_{n, k}^{(m)}\left(r_{1}, \ldots, r_{m}, r_{m+1}-1, \ldots, r_{m+2}, \ldots, r_{n-k m+1}\right)\right. \\
& \left.\quad-\sum_{i=m+1}^{n-k m+1}\left(r_{i}+1\right) b_{n, k}^{(m)}\left(r_{1}, \ldots, r_{m}-1, \ldots, r_{i}+1, r_{i+1}-1, \ldots, r_{n-k m+1}\right)\right) .
\end{aligned}
$$

Полагая $m=1$ в соотношении (20) получаем соотношение (12). 
Отметим, что числа Стирлинга и Лаха могут быть представлены через коэффициенты $b_{n, k}^{(m)}(r)$ в виде

$$
\begin{aligned}
S(n, k) & =\sum_{n, k} b_{n, k}(r) \\
|s(n, k)| & =\sum_{n, k} b_{n, k}(r) \prod_{i=1}^{n-k+1}((i-1) !)^{r_{i}}, \\
|L(n, k)| & =\sum_{n, k} b_{n, k}(r) \prod_{i=1}^{n-k+1}(i !)^{r_{i}}, \quad n \geqslant 2, \quad 1 \leqslant k \leqslant n-1 .
\end{aligned}
$$

\section{4. Полиномы Тушара и $P$-полиномы}

\section{1. $\quad$-п-полиномы Тушара}

Полиномы от переменных $x_{i}$ и $y_{i}, i \geqslant 1$, которые мы будем обозначать

$$
T_{n, k}(x, y)=T_{n, k}\left(x_{1}, \ldots ; y_{1}, \ldots\right), \quad n, k \geqslant 1, \quad k \leqslant n
$$

и в дальнейшем называть $T$-полиномами Тушара, впервые рассматривались Тушаром в [9]. Производящая функция полиномов Тушара имеет вид

$$
\sum_{n=k}^{\infty} T_{n, k}(x, y) \frac{t^{n}}{n !}=(x(t))^{k} \frac{e^{y(t)}}{k !}
$$

где

$$
x(t)=\sum_{i=1}^{\infty} x_{i} \frac{t^{i}}{i !}, \quad y(t)=\sum_{i=1}^{\infty} y_{i} \frac{t^{i}}{i !} .
$$

Известен явный вид этих полиномов (см., например, [10]):

$$
T_{n, k}(x, y)=n ! \sum_{(n ; k)} \prod_{i=1}^{n} x_{i}^{r_{i}} y_{i}^{s_{i}}\left(r_{i} ! s_{i} !(i !)^{r_{i}+s_{i}}\right)^{-1}
$$

где $T_{0,0}(x, y)=1$ и суммирование ведется по всем наборам целых неотрицательных чисел $r_{i}, s_{i}, 1 \leqslant i \leqslant n$, таким, что

$$
\sum_{i=1}^{n} r_{i}=k, \quad \sum_{i=1}^{n} i\left(r_{i}+s_{i}\right)=n .
$$

Непосредственно из (22) следует, что

$$
T_{n, 0}(x, y)=Y_{n}(y)
$$

где $Y_{n}(y)=Y_{n}\left(y_{1}, \ldots, y_{n}\right)$ - хорошо известные полиномы Белла (см., например, [2]), и

$$
T_{n, n}(x, y)=x_{1}^{n}=A_{n n}(x)
$$


Из сопоставления формул (1) и (21) или (3) и (22) видно, что

$$
T_{n, k}(x, 0)=A_{n, k}(x) \text {. }
$$

Полиномы Тушара удовлетворяют системе дифференциально-разностных соотношений [12]

$$
T_{n, k}(x, y)=x_{1} T_{n-1, k-1}(x, y)+\left(\mathscr{D}_{x}+\mathscr{D}_{y}+y_{1}\right) T_{n-1, k}(x, y), \quad n, k \geqslant 1, \quad k \leqslant n .
$$

Соотношение (22) можно переписать в виде

$$
T_{n, k}(x, y)=\sum_{(n ; k)} t_{n, k}(r, s) \prod_{i=1}^{n} x_{i}^{r_{i}} y_{i}^{s_{i}}
$$

где

$$
t_{n, k}(r, s)=n ! \prod_{i=1}^{n}\left(r_{i} ! s_{i} !(i !)^{r_{i}+s_{i}}\right)^{-1} .
$$

Сравнивая в в левой и правой части формулы (23) коэффициенты при произведении $\prod_{i=1}^{n} x_{i}^{r_{i}} y_{i}^{s_{i}}$, получаем рекуррентное соотношение

$$
\begin{aligned}
& t_{n, k}(r, s)=t_{n-1, k-1}\left(r_{1}-1, r_{2}, \ldots, r_{n} ; s\right) \\
&+\sum_{i=1}^{n-k}\left(r_{i}+1\right) t_{n-1, k}\left(r_{1}, \ldots, r_{i-1}, r_{i}+1, r_{i+1}-1, r_{i+2}, \ldots, r_{n} ; s\right) \\
&+t_{n-1, k}\left(r ; s_{1}-1, s_{2}, \ldots, s_{n}\right) \\
&+\sum_{i=1}^{n-k}\left(s_{i}+1\right) t_{n-1, k}\left(r ; s_{1}, \ldots, s_{i-1}, s_{i}+1, s_{i+1}-1, s_{i+2}, \ldots, s_{n}\right) .
\end{aligned}
$$

Отметим, что числа Стирлинга и Лаха могут быть представлены через коэффициенты $t_{n, k}(r, s)$ в следующем виде:

$$
\begin{aligned}
S(n, k) & =\sum_{n, k} t_{n, k}(r, s) \prod_{i=1}^{n} s_{i} !(i !)^{s_{i}} \\
|s(n, k)| & =\sum_{n, k} t_{n, k}(r, s) \prod_{i=1}^{n} s_{i} !(i !)^{s_{i}}((i-1) !)^{r_{i}}, \\
L(n, k) & =\sum_{n, k} t_{n, k}(r, s) \prod_{i=1}^{n} s_{i} !(i !)^{r_{i}+s_{i}}, \quad n \geqslant 2, \quad 1 \leqslant k \leqslant n-1 .
\end{aligned}
$$

\section{2. Р-полиномы}

Полиномы от $x_{i}$ и $y_{i}, i \geqslant 1$, обозначаемые

$$
P_{n, k}(x, y)=P_{n, k}\left(x_{1}, x_{2}, \ldots ; y_{1}, y_{2}, \ldots\right), \quad n, k \geqslant 1, \quad k \leqslant n,
$$

и называемые $P$-полиномами, впервые рассматривались авторами этой статьи в [14] и могут быть определены производящей функцией

$$
\sum_{n=k}^{\infty} P_{n, k}(x, y) \frac{u^{n}}{n !}=\frac{\tilde{x}(u)^{k}}{k !} e^{-y(\tilde{x}(u))}, \quad k \geqslant 0 .
$$


Разлагая в ряд правую часть (25) и приравнивая коэффициенты при $u^{n} / n !$, получаем явную формулу для $P$-полиномов

$$
\begin{aligned}
P_{n, k}(x, y) & =\frac{(-1)^{n-k}}{k ! x_{1}^{2 n-k}} \sum_{(2 n-2 k, n-k)}(-1)^{r_{1}+s_{1}+\ldots+s_{n}} r_{1} ! \\
& \times\left(2 n-k-r_{1}-1\right) !\left(\sum_{i=1}^{n} i s_{i}+k\right) \prod_{i=1}^{n} x_{i}^{r_{i}} y_{i}^{s_{i}}\left(r_{i} ! s_{i} !(i !)^{r_{i}+s_{i}}\right)^{-1}, \quad 1 \leqslant k \leqslant n,
\end{aligned}
$$

где $P_{n, n}(x, y)=x_{1}{ }^{-n}, n \geqslant 1$, и суммирование ведется по всем наборам цельх неотрицательных чисел $r_{i}, s_{i}, 1 \leqslant i \leqslant n$, таким, что

$$
\sum_{i=1}^{n} r_{i}=n-k, \quad \sum_{i=1}^{n} i\left(r_{i}+s_{i}\right)=2 n-2 k
$$

Из (4) и (9) или (8) и (12) следует, что

$$
P_{n, k}(x, 0)=B_{n, k}(x),
$$

то есть $P$-полиномы являются обобщениями $B$-полиномов Платонова.

Известно (см. [14]), что $P$-полиномы удовлетворяют системе дифференциальноразностных соотношений

$$
x_{1} P_{n, k}(x, y)=P_{n-1, k-1}(x, y)+\left(\mathscr{D}_{x}+\mathscr{D}_{y}-y_{1}\right) P_{n-1, k}(x, y), \quad 1 \leqslant k \leqslant n
$$

и связаны с $T$-полиномами соотношениями квазиортогональности

$$
\begin{aligned}
& \sum_{i=k}^{n} T_{n, i}(x, y) P_{i, k}(x, y)=\delta_{n, k}, \\
& \sum_{i=k}^{n} P_{n, i}(x, y) T_{i, k}(x, y)=\delta_{n, k}, \quad 1 \leqslant k \leqslant n .
\end{aligned}
$$

Вводя нормирующие множители $(-1)^{n-k} x_{1}^{2 n-k}$, получим полиномы от всех переменных $x_{i}, i \geqslant 1$

$$
P_{n, k}^{\prime}(x, y)=(-1)^{n-k} x_{1}^{2 n-k} P_{n, k}(x, y)=\sum_{(2 n-2 k, n-k)} p_{n, k}(r, s) \prod_{i=1}^{n} x_{i}^{r_{i}} y_{i}^{s_{i}}
$$

где

$$
p_{n, k}(r)=(-1)^{r_{1}+s_{1}+\ldots+s_{n}} \frac{r_{1} !}{k !}\left(2 n-k-r_{1}-1\right) !\left(\sum_{i=1}^{n} i r_{i}+k\right) \prod_{i=1}^{n}\left(r_{i} ! s_{i} !(i !)^{r_{i}+s_{i}}\right)^{-1}
$$

Сравнивая в левой и правой частях (26) коэффициенты при произведении 
$\prod_{i=1}^{n} x_{i}^{r_{i}} y_{i}^{s_{i}}$, получаем рекуррентное соотношение

$$
\begin{aligned}
& p_{n, k}(r, s)=p_{n-1, k-1}(r, s)+(k-2 n+2) p_{n-1, k}\left(r_{1}, r_{2}-1, r_{3}, \ldots, r_{n} ; s\right) \\
& \quad-\sum_{i=1}^{n}\left(r_{i}+1\right) p_{n-1, k}\left(r_{1}-1, r_{2}, \ldots, r_{i-1}, r_{i}+1, r_{i+1}-1, r_{i+2}, \ldots, r_{n} ; s\right) \\
& \quad+p_{n-1, k}\left(r_{1}-1, r_{2}, \ldots, r_{n} ; s_{1}-1, s_{2}, \ldots, s_{n}\right) \\
& \quad-\sum_{i=1}^{n}\left(s_{i}+1\right) p_{n-1, k}\left(r_{1}-1, r_{2}, \ldots, r_{n} ; s_{1}, \ldots, s_{i-1}, s_{i}+1, s_{i+1}-1, s_{i+2}, \ldots, s_{n}\right) .
\end{aligned}
$$

Отметим, что числа Стирлинга и Лаха выражаются через коэффициенты $p_{n, k}(r, s)$ в виде

$$
\begin{aligned}
S(n, k) & =\sum_{2 n-2 k, n-k} p_{n, k}(r, s)(-1)^{s_{1}+\ldots+s_{n}} k\left(\sum_{i=1}^{n} i r_{i}+k\right)^{-1} \prod_{i=1}^{n} s_{i} !(i !)^{s_{i}}((i-1) !)^{r_{i}}, \\
|s(n, k)| & =\sum_{2 n-2 k, n-k} p_{n, k}(r, s)(-1)^{s_{1}+\ldots+s_{n}} k\left(\sum_{i=1}^{n} i r_{i}+k\right)^{-1} \prod_{i=1}^{n} s_{i} !(i !)^{s_{i}} \\
|L(n, k)| & =\sum_{2 n-2 k, n-k} p_{n, k}(r, s)(-1)^{s_{1}+\ldots+s_{n}} k\left(\sum_{i=1}^{n} i r_{i}+k\right)^{-1} \prod_{i=1}^{n} s_{i} !(i !)^{r_{i}+s_{i}}
\end{aligned}
$$

где $n \geqslant 2,1 \leqslant k \leqslant n-1$.

\section{3. $C$-полиномы Тушара}

Цикловой индикатор симметрической группы степени $n$ равен

$$
C_{n}(t)=C_{n}\left(t_{1}, \ldots, t_{n}\right)=n ! \sum \prod_{i=1}^{n} t_{i}^{k_{i}}\left(k_{i} ! i^{k_{i}}\right)^{-1}
$$

(см., например, [2]), где $t=\left(t_{1}, \ldots, t_{n}\right)$, суммирование ведется по всем наборам цельх неотрицательных чисел $k_{i}, 1 \leqslant i \leqslant n$, таким, что $k_{1}+2 k_{2}+\ldots+n k_{n}=n$ и $C_{0}=1$.

Цикловые индикаторы связаны с полиномами Белла соотношениями

$$
C_{n}(t)=Y_{n}\left(1 ; t_{1}, t_{2}, 2 ! t_{3}, \ldots,(n-1) ! t_{n}\right)
$$

(см., например, [2]).

В [11] при помощи соотношений

$$
\begin{aligned}
C_{n, k}(x, y) & =C_{n, k}\left(x_{1}, \ldots ; y_{1}, \ldots\right) \\
& =\left(\begin{array}{l}
n \\
k
\end{array}\right) C_{k}(x) C_{n-k}(y), \quad 0 \leqslant k \leqslant n, \quad n \geqslant 1,
\end{aligned}
$$

введены полиномы $C_{n, k}(x, y)$, которые мы будем назвать $C$-полиномами Тушара.

$T$ - и $C$-полиномы связаны между собой соотношением (см. [13])

$$
\sum_{k=0}^{n} C_{n, k}(x, y)=\sum_{k=0}^{n} T_{n, k}\left(x^{*}, y^{*}\right)
$$


где $x_{i}^{*}=(i-1) ! x_{i}, y_{i}^{*}=(i-1) ! y_{i}$.

Из (28) и (30) следует, что

$$
C_{n, k}(x, y)=n ! \sum \prod_{i=1}^{n} x_{i}^{r_{i}} y_{i}^{s_{i}}\left(r_{i} ! s_{i} ! i^{r_{i}+s_{i}}\right)^{-1}
$$

где $C_{0,0}(x, y)=1$ и суммирование ведется по всем наборам целых неотрицательных чисел $r_{i}, s_{i}, 1 \leqslant i \leqslant n$, таким, что

$$
\sum_{i=1}^{n} i r_{i}=k, \quad \sum_{i=1}^{n} i s_{i}=n-k .
$$

Из соотношений (29) и (30) следует, что

$$
\sum_{n=k}^{\infty} C_{n, k}(x, y) \frac{u^{n}}{n !}=e^{u \varphi(t)}
$$

где

$$
\varphi(t)=\sum_{i=1}^{\infty} \frac{x_{i}+y_{i}}{i} t^{i}
$$

Теорема 1. Для C-полиномов справедливъ соотношения

$$
\begin{aligned}
\frac{\partial}{\partial x_{i}} C_{n, k}(x, y) & =\left(\begin{array}{c}
n \\
i
\end{array}\right)(i-1) ! C_{n-i, k-i}(x, y), \quad k, i \geqslant 1, \quad n \geqslant k, \\
\frac{\partial}{\partial y_{i}} C_{n, k}(x, y) & =\left(\begin{array}{c}
n \\
i
\end{array}\right)(i-1) ! C_{n-i, k}(x, y), \quad k \geqslant 0, \quad i \geqslant 1, \quad n \geqslant k+i .
\end{aligned}
$$

Доказательство. Докажем соотношение (32). Известно [6], что

$$
r \frac{\partial}{\partial t_{r}} C_{n}(t)=(n)_{r} C_{n-r}(t) .
$$

Продифференцировав соотношение (30) по $x_{i}$, с учетом (34) получаем, что

$$
\begin{aligned}
\frac{\partial}{\partial x_{i}} C_{n, k}(x, y) & =\left(\begin{array}{l}
n \\
k
\end{array}\right) \frac{\partial}{\partial x_{i}} C_{k}(x) C_{n-k}(y)=\left(\begin{array}{l}
n \\
k
\end{array}\right) \frac{1}{i}(k)_{i} C_{k-i}(x) C_{n-k}(y) \\
& =\frac{n ! k !}{k !(n-k) !(k-i) ! i} C_{k-i}(x) C_{n-k}(y) \\
& =\frac{n !(n-i) !(i-1) !}{(n-k) !(k-i) ! i(n-i) !(i-1) !} C_{k-i}(x) C_{n-k}(y) \\
& =\left(\begin{array}{c}
n-i \\
k-i
\end{array}\right)\left(\begin{array}{c}
n \\
i
\end{array}\right)(i-1) ! C_{k-i}(x) C_{n-k}(y)=\left(\begin{array}{c}
n \\
i
\end{array}\right)(i-1) ! C_{n-i, k-i}(x, y) .
\end{aligned}
$$

Соотношение (33) доказывается аналогично.

Теорема 2. При $n, k \geqslant 1$ справедливы соотношения

$$
\begin{aligned}
& C_{n, k}(x, y)=\sum_{i=0}^{k-1} \frac{n !}{k(n-i-1) !} x_{i+1} C_{n-i-1, k-i-1}(x, y), \\
& C_{n, k}(x, y)=\sum_{i=0}^{n-k-1} \frac{n !}{(n-k)(n-i-1) !} y_{i+1} C_{n-i-1, k}(x, y) .
\end{aligned}
$$


Доказательство. Докажем соотношение (35). Известно [2], что

$$
C_{n+1}(t)=\sum_{i=0}^{n}(n)_{i} t_{i+1} C_{n-i}(t) .
$$

Из (30) с учетом соотношения (37) следует, что

$$
\begin{aligned}
C_{n, k}(x, y) & =\left(\begin{array}{l}
n \\
k
\end{array}\right) \sum_{i=0}^{k-1}(k-1)_{i} x_{i+1} C_{k-i-1}(x) C_{n-k}(y) \\
& =\sum_{i=0}^{k-1} \frac{n !(k-1) !}{k !(n-k) !(k-i-1) !} x_{i+1} C_{k-i-1}(x) C_{n-k}(y) \\
& =\sum_{i=0}^{k-1} \frac{n !(n-i-1) !}{k(n-k) !(k-i-1) !(n-i-1) !} x_{i+1} C_{k-i-1}(x) C_{n-k}(y) \\
& =\sum_{i=0}^{k-1}\left(\begin{array}{l}
n-i-1 \\
k-i-1
\end{array}\right) \frac{n !}{k(n-i-1) !} x_{i+1} C_{k-i-1}(x) C_{n-k}(y) \\
& =\sum_{i=0}^{k-1} \frac{n !}{k(n-i-1) !} x_{i+1} C_{n-i-1, k-i-1}(x, y) .
\end{aligned}
$$

Соотношение (36) доказывается аналогично.

Введем обозначения

$$
\mathscr{D}_{x}(k)=\sum_{i=1}^{k} i x_{i+1} \frac{\partial}{\partial x_{i}}, \quad \mathscr{D}_{y}(n, k)=\sum_{i=1}^{n-k} i y_{i+1} \frac{\partial}{\partial y_{i}} .
$$

Теорема 3. Для C-полиномов справедливы соотношения

$$
C_{n, k}(x, y)=\left(x_{1}+\mathscr{D}_{x}(k-1)\right) C_{n-1, k-1}(x, y)+\left(y_{1}+\mathscr{D}_{y}(n, k)\right) C_{n-1, k}(x, y),
$$

где $k \geqslant 1, n \geqslant k+1$.

Доказательство. Из (35) с учетом соотношения (32) получаем, что

$$
\begin{aligned}
C_{n, k}(x, y) & =\frac{n}{k} x_{1} C_{n-1, k-1}(x, y)+\sum_{i=1}^{k-1} \frac{n !}{k(n-i-1) !} x_{i+1} C_{n-i-1, k-i-1}(x, y) \\
& =\frac{n}{k} x_{1} C_{n-1, k-1}(x, y)+\sum_{i=1}^{k-1} i \frac{n}{k} x_{i+1} \frac{\partial}{\partial x_{i}} C_{n-1, k-1}(x, y)
\end{aligned}
$$

или

$$
\frac{k}{n} C_{n, k}(x, y)=x_{1} C_{n-1, k-1}(x, y)+\sum_{i=1}^{k-1} i x_{i+1} \frac{\partial}{\partial x_{i}} C_{n-1, k-1}(x, y)
$$


Из (36) с учетом формулы (31) следует, что

$$
\begin{aligned}
C_{n, k}(x, y) & =\frac{n}{n-k} y_{1} C_{n-1, k}(x, y)+\sum_{i=1}^{n-k-1} \frac{n !}{(n-k)(n-i-1) !} y_{i+1} C_{n-i-1, k}(x, y) \\
& =\frac{n}{n-k} y_{1} C_{n-1, k}(x, y)+\sum_{i=1}^{n-k-1} \frac{n}{n-k} i y_{i+1} \frac{\partial}{\partial y_{i}} C_{n-i, k}(x, y)
\end{aligned}
$$

или

$$
\frac{n-k}{n} C_{n, k}(x, y)=y_{1} C_{n-1, k}(x, y)+\sum_{i=1}^{n-k-1} i y_{i+1} \frac{\partial}{\partial y_{i}} C_{n-i, k}(x, y) .
$$

Из (39) и (40) следует, что

$$
C_{n, k}(x, y)=\left(x_{1}+\mathscr{D}_{x}(k-1)\right) C_{n-1, k-1}(x, y)+\left(y_{1}+\mathscr{D}_{y}(n-k)\right) C_{n-1, k}(x, y) .
$$

Теорема доказана.

Замечание 1. Если соотношение (38) преобразовать с помощью формул (32) и (33), то получим рекуррентное соотношение для полиномов $C_{n, k}(x, y)$, приведенное в [13].

Соотношение (31) перепишем в виде

$$
C_{n, k}(x, y)=\sum c_{n, k}(r, s) \prod_{i=1}^{n} x_{i}^{r_{i}} y_{i}^{s_{i}}
$$

где

$$
c_{n, k}(r, s)=n ! \prod_{i=1}^{n}\left(r_{i} ! s_{i} ! i^{r_{i}+s_{i}}\right)^{-1}
$$

Как видно из (41), коэффициент $c_{n, k}(r, s)$ можно интерпретировать как число подстановок степени $n$ с $r$ циклами, обладающими специальными свойствами.

Сравнивая в формуле (38) коэффициенты в левой и правой части при произведении $\prod_{i=1}^{n} x_{i}^{r_{i}} y_{i}^{s_{i}}$, находим, что справедливо рекуррентное соотношение

$$
\begin{aligned}
c_{n, k}(r, s)=c_{n-1, k-1}\left(r_{1}-1, r_{2}, \ldots, r_{n} ; s\right)+c_{n-1, k}\left(r ; s_{1}-1, s_{2}, \ldots, s_{n}\right) \\
\quad+\sum_{i=1}^{k-1}\left(r_{i}+1\right) i c_{n-1, k-1}\left(r_{1}, \ldots, r_{i-1}, r_{i}+1, r_{i+1}-1, r_{i+2}, \ldots, r_{n} ; s\right) \\
\quad+\sum_{i=1}^{n-k+1}\left(s_{i}+1\right) i c_{n-1, k}\left(r ; s_{1}, \ldots, s_{i-1}, s_{i}+1, s_{i+1}-1, s_{i+2}, \ldots, s_{n}\right) .
\end{aligned}
$$

Отметим, что числа Стирлинга и Лаха могут быть представлены через коэффициенты $c_{n, k}(r, s)$ в виде

$$
\begin{gathered}
S(n, k)=\sum_{n, k} c_{n, k}(r, s) \prod_{i=1}^{n-k+1} s_{i} ! i^{s_{i}}((i-1) !)^{-r_{i}} \\
|s(n, k)|=\sum_{n, k} c_{n, k}(r, s) \prod_{i=1}^{n-k+1} s_{i} ! i^{s_{i}}, \quad|L(n, k)|=\sum_{n, k} c_{n, k}(r, s) \prod_{i=1}^{n-k+1} s_{i} ! i^{r_{i}+s_{i}}
\end{gathered}
$$

где $n \geqslant 2,1 \leqslant k \leqslant n-1$. 


\section{Список литературы}

1. Bell E. F., Exponential polynomials. Ann. Math. (1934) 35, 258-277.

2. Риордан Дж., Введение в комбинаторнъй анализ. ИЛ, Москва, 1963.

3. Кузьмин О. В., Рекуррентные соотношения и перечислительные интерпретации некоторых комбинаторных чисел и полиномов. Дискретная математика (1994) 6, №3, $39-49$.

4. Жуков В. Д., Рекуррентные формулы для обобщенных $A$ - и $B$-полиномов. В кн.: Исследования по геомагнетизму, аэрономии и физике Солнца. Наука, Москва, 1983, с. 192 197.

5. Comtet L., Advanced Combinatorics. Reidel, Dordrecht, 1974.

6. Howard F. T., Bell polynomials and degenerate Stirling numbers. Rend. Sem. Math. Univ. Padova. (1980) 61, 203-219.

7. Селиванов Б. И., Комбинаторный подход к формуле обращения Бюрмана-Лагранжа. Комбинаторнъй и асимптотический аяализ. Изд-во Красноярского унив., Красноярск, 1977, 153-169.

8. Кузьмин О. В., Построение обобщенных $A$ - и $B$-полиномов в пространстве отображений. Методы дискретного анализа в теории графов и сложности. Институт Матем. СО РАН, Новосибирск, 1992, вып. 52, 66-76.

9. Touchrd J., Sur les cycles des substitutious. Acta Math. (1939) 70, 243-279.

10. Chrysaphinou O., On Touchard polynomials. Discrete Math. (1985) 54, 143-152.

11. Charalambides Ch. A., Chrysaphinou O., Partition polynomials in fluctuation theory. Math. Nachr. (1982) 106, 89-100.

12. Кузьмин О. В., Леонова О. В., О полиномах Тушара. Асимптотические и перечислителънъе задачи комбинаторного анализа. Изд-во Иркутского унив., Иркутск, 1997, 101-109.

13. Кузьмин О. В., Леонова О. В., Полиномы Тушара и их приложения. Дискретяая математика (2000) 12, №3, 60-71.

14. Кузьмин О. В., Леонова О. В., Полиномы Тушара и им квазиортогональные. Oптимизачия, управление, интеллект. ИДСТУ СО РАН, Иркутск, 1999, вып. 3, 218-227.

15. Докин В. Н., Жуков В. Д., Колокольникова Н. А., Кузьмин О. В., Платонов М. Л., Комбинаторные числа и полиномы в моделях дискретных распределений. Изд-во Иркутского унив., Иркутск, 1990.

16. Платонов М. Л., Комбинаторные числа класса отображений. Комбинаторный и асимптотический анализ. Изд-во Красноярского унив., Красноярск, 1975, 81-85.

17. Платонов М. Л., Комбинаторные числа класса отображений и их приложения. Наука, Москва, 1979.

18. Кузьмин О. В., Обобщеняъе пирамиды Паскаля и их приложения. Наука, Новосибирск, 2000.

Статья поступила 30.01.2001. 